\title{
A Lagrangian meshless finite element method applied to fluid-structure interaction problems
}

\author{
S.R. Idelsohn ${ }^{\mathrm{a}, \mathrm{b}, *}$, E. Oñate ${ }^{\mathrm{b}}$, F. Del Pin ${ }^{\mathrm{a}}$ \\ a International Center for Computational Methods in Engineering (CIMEC), Universidad Nacional del Litoral and CONICET, \\ Guemes 3450, 3000 Santa Fe, Argentina \\ ${ }^{\mathrm{b}}$ International Center for Numerical Methods in Engineering (CIMNE), Universidad Politécnica de Cataluña, \\ Edificio C1 Campus Nord, 08032 Barcelona, Spain
}

\begin{abstract}
A method is presented for the solution of the incompressible fluid flow equations using a Lagrangian formulation. The interpolation functions are those used in the meshless finite element method and the time integration is introduced in a semi-implicit way by a fractional step method. Classical stabilization terms used in the momentum equations are unnecessary due to the lack of convective terms in the Lagrangian formulation. Furthermore, the Lagrangian formulation simplifies the connections with fixed or moving solid structures, thus providing a very easy way to solve fluidstructure interaction problems.
\end{abstract}

(C) 2003 Elsevier Science Ltd. All rights reserved.

Keywords: Fluid-structure interaction; Particle methods; Lagrange formulations; Incompressible fluid flows; Meshless methods; Finite element method

\section{Introduction}

Over the last 20 years, computer simulation of incompressible fluid flow has been based on the Eulerian formulation of the fluid mechanics equations. However, it is still difficult to analyze problems in which the shape of the interface changes continuously or in fluid-structure interactions (FSI) with free-surfaces where complicated contact problems are involved.

More recently, particle methods in which each fluid particle is followed in a Lagrangian manner have been used [1-4]. The first ideas on this approach were proposed by Gingold and Monaghan [1] for the treatment of astrophysical hydrodynamic problems with the so

\footnotetext{
${ }^{*}$ Corresponding author. Address: International Center for Computational Methods in Engineering (CIMEC), Universidad Nacional del Litoral and CONICET, Guemes 3450, 3000 Santa $\mathrm{Fe}$, Argentina.

E-mail addresses: sergio@ceride.gov.ar (S.R. Idelsohn), onate@cimne.upc.es (E. Oñate).
}

called smooth particle hydrodynamics method (SPH). This method was later generalized to fluid mechanic problems [2-4]. Kernel approximations are used in the SPH method to interpolate the unknowns.

On the other hand, a family of methods called meshless methods have been developed both for structural $[5,6]$ and fluid mechanics problems [8-10]. All these methods use the idea of a polynomial interpolant that fits a number of points minimizing the distance between the interpolated function and the value of the unknown point. These ideas were proposed first by Nayroles et al. [7], they were later used in structural mechanics by Belytschko et al. [5] and in fluid mechanics problems by Oñate and co-workers [8-10]. In a previous paper, [11] the authors presented the numerical solution for the fluid mechanics equations using a Lagrangian formulation and a meshless method called the finite point method. Lately, the meshless ideas were generalized to take into account the finite element type approximations in order to obtain the same computing time in mesh generation as in the evaluation of the meshless connectivities $[12,13]$. This method was called the meshless 
finite element method (MFEM) and uses the extended Delaunay tessellation (EDT) [14] to build the mesh in a computing time which is linear with the number of nodal points.

In this paper, new ideas and results for the solution of a particle method in the field of FSI using the MFEM are presented. A more general formulation is used in which all the classical advantages of the FEM for the evaluation of the unknown functions and derivatives are preserved.

Different strategies have been proposed to solve FSI problems. The selection of the most effective approach depends largely on the nature of the problem to be analyzed [15]. Depending on the degree of coupling between the equations for the fluid and the structure, two cases can be distinguished. The first one occurs when there is a strong coupling between the fluid flow and the elastic deformation of the structure [15-17]. The second case occurs when there is a weak interaction between the fluid and the rigid deformation of the structure. In the latter, the solid must undergo large rigid displacements interacting with the fluid. This is the case for instance of sea-keeping in ship hydrodynamics, rotating turbines, mills, and other engines with a moving solid inside a flutid. Both cases of FSI are more easily studied with a Lagrangian formulation of can be seen as a solid with

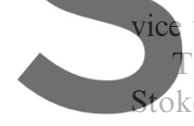

he Lagrangian fluid flow problem will be revisd
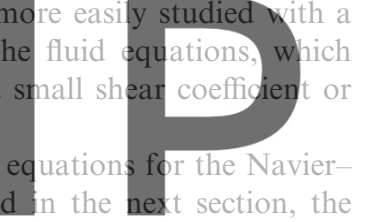

MFEM will be summarized in Appendix A and both Registechniques will be used to solve some ES alioblems for

\section{Governing equations}

The mass and momentum conservation equations can be written in a Lagrangian formulations as mass conservation:

$\frac{\mathrm{D} \rho}{\mathrm{D} t}+\rho \frac{\partial u_{i}}{\partial x_{i}}=0$

momentum conservation:

$\rho \frac{\mathrm{D} u_{i}}{\mathrm{D} t}=-\frac{\partial}{\partial x_{i}} p+\frac{\partial}{\partial x_{j}} \tau_{i j}+\rho f_{i}$

where $\rho$ is the density $u_{i}$ are the Cartesian components of the velocity field, $p$ the pressure, $\tau_{i j}$ the deviator stress tensor, $f_{i}$ the source term (normally the gravity) and $\mathrm{D} \phi / \mathrm{D} t$ represents the total or material time derivative of a function $\phi$.

For Newtonian fluids the stress tensor $\tau_{i j}$ may be expressed as a function of the velocity field through the viscosity $\mu$ by $\tau_{i j}=\mu\left(\frac{\partial u_{i}}{\partial x_{j}}+\frac{\partial u_{j}}{\partial x_{i}}-\frac{2}{3} \frac{\partial u_{l}}{\partial x_{l}} \delta_{i j}\right)$

For near incompressible flows $\left(\frac{\partial u_{i}}{\partial x_{i}} \ll \frac{\partial u_{k}}{\partial x_{l}}\right)$ the term

$\frac{2 \mu}{3} \frac{\partial u_{i}}{\partial x_{i}} \approx 0$

and it may be neglected in Eq. (3). Then

$\tau_{i j} \approx \mu\left(\frac{\partial u_{i}}{\partial x_{j}}+\frac{\partial u_{j}}{\partial x_{i}}\right)$

In the same way, the term $\frac{\partial}{\partial x_{j}} \tau_{i j}$ in the momentum equations may be simplified for slow incompressible flows as
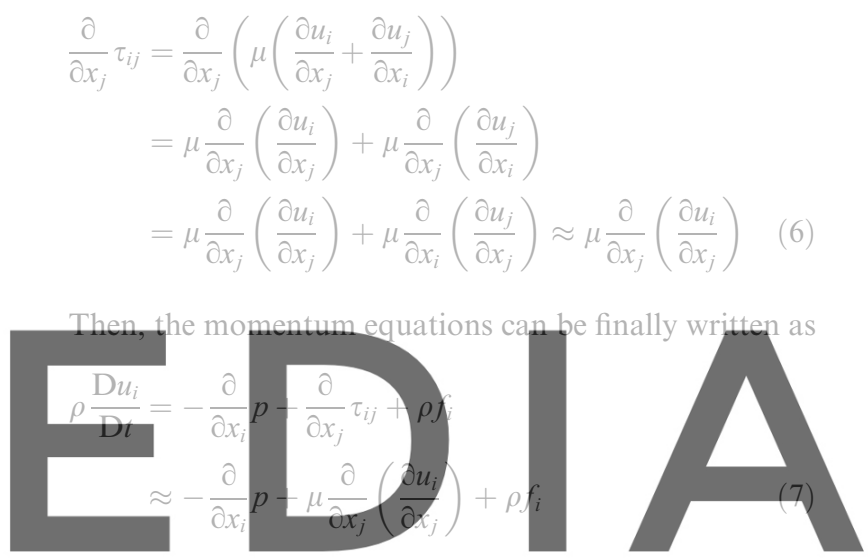

Boundary conditions

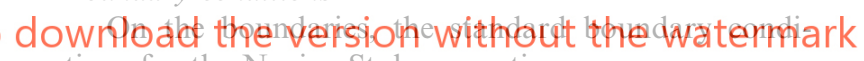

tions for the Navier-Stokes equations are

$\tau_{i j} v_{j}-p v_{i}=\bar{\sigma}_{n i}$ on $\Gamma_{\sigma}$

$u_{i} v_{i}=\bar{u}_{n} \quad$ on $\Gamma_{n}$

$u_{i} \zeta_{i}=\bar{u}_{t} \quad$ on $\Gamma_{t}$

where $v_{i}$ and $\zeta_{i}$ are the components of the normal and tangent vector to the boundary.

\section{The time splitting}

The time integration of Eqs. (7) and (8) presents some difficulties when the fluid is incompressible or nearly incompressible. In this case, explicit time steps cannot be used. Even when using an implicit time integration scheme, incompressibility introduces some wiggles in the pressure solution which must be stabilized. To overcome these difficulties, a fractional step method has been proposed [18] which consists in splitting each time step in 2 steps as follows. 
Split of the momentum equations

$$
\begin{aligned}
\frac{\mathrm{D} u_{i}}{\mathrm{D} t} & \approx \frac{u_{i}^{n+1}-u_{i}^{n}}{\Delta t}=\frac{u_{i}^{n+1}-u_{i}^{*}+u_{i}^{*}-u_{i}^{n}}{\Delta t} \\
& =\left(-\frac{1}{\rho} \frac{\partial}{\partial x_{i}} p+\frac{1}{\rho} \frac{\partial \tau_{i j}}{\partial x_{j}}+f_{i}\right)^{n+\theta}
\end{aligned}
$$

where $\Delta t=t^{n+1}-t^{n}$ is the time step; $u_{i}^{n}=u_{i}\left(x^{n}, t^{n}\right)$; $u_{i}^{n+1}=u_{i}\left(x^{n+1}, t^{n+1}\right)$ and $u_{i}^{*}$ are fictitious variables defined by the split
(A) $u_{i}^{*}=u_{i}^{n}+f_{i} \Delta t-\frac{\Delta t}{\rho} \frac{\partial}{\partial x_{i}} \gamma p^{n}+\frac{\Delta t}{\rho} \frac{\partial}{\partial x_{j}} \tau_{i j}^{n}$
(C) $u_{i}^{n+1}=u_{i}^{*}-\frac{\Delta t}{\rho} \frac{\partial}{\partial x_{i}}\left(p^{n+1}-\gamma p^{n}\right)$

where $\gamma$ is parameter equal to zero or one defining a first or second order split, respectively [18].

Split of the mass conservation equations
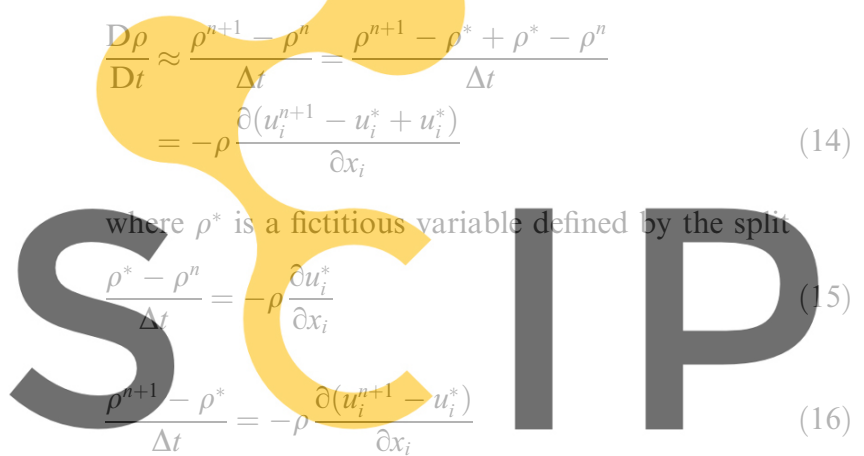

Regisferpłodrefiretiongt https//www.scipedia.com to From Eqs. (13) and (16) the coupled mass-momentum equation becomes

(B)

$$
\frac{\rho^{n+1}-\rho *}{\Delta t^{2}}=\frac{\partial^{2}}{\partial x_{i}^{2}}\left(p^{n+1}-\gamma p^{n}\right)
$$

Taking into account Eq. (15) above expression can be written as

(B) $\frac{\rho^{n+1}-\rho^{n}}{\Delta t^{2}}+\frac{\rho}{\Delta t} \frac{\partial u_{i}^{*}}{\partial x_{i}}=\frac{\partial^{2}}{\partial x_{i}^{2}}\left(p^{n+1}-\gamma p^{n}\right)$

\section{Incompressibility conditions}

The simplest way to introduce the incompressibility condition is to write

$\rho^{n+1}=\rho^{n}=\rho^{0}=\rho$

Then, the first term of Eq. (18) disappears. Nevertheless, in a Lagrangian formulation it is better to evaluate this term in order to avoid possible numerical errors at each time step. The incompressibility condition is introduced by imposing that at time step $t^{n+1}$ the density must be equal to the initial one, i.e. $\rho^{n+1}=\rho^{0}=\rho$

Due to numerical errors the density $\rho^{n}$ is not necessarily equal to $\rho_{0}$ and it must be updated at each time step. A different way to evaluate $\rho^{n}$ will be explained in Section 7. Eq. (18) is finally written as

(B) $\frac{\rho^{0}-\rho^{n}}{\Delta t^{2}}+\frac{\rho}{\Delta t} \frac{\partial u_{i}^{*}}{\partial x_{i}}=\frac{\partial^{2}}{\partial x_{i}^{2}}\left(p^{n+1}-\gamma p^{n}\right)$

Then, the total time step may be described as follows: starting with the known value $u^{n}$ and $p^{n}$ from the previous time increment, the computation of the new velocities and the pressure involves the following five steps.

(I) Evaluate the $u^{*}$ velocity from (12).

(II) Evaluate the new density $\rho^{n}$ (see Section 7).

(III) Evaluate the pressure $p^{n+1}$ solving the Laplacian Eq. (21).

(IV) Evaluate the velocity $u^{n+1}$ using (13).

(V) Move the particles to the $x^{n+1}$ position.

\section{Spatial discretization}

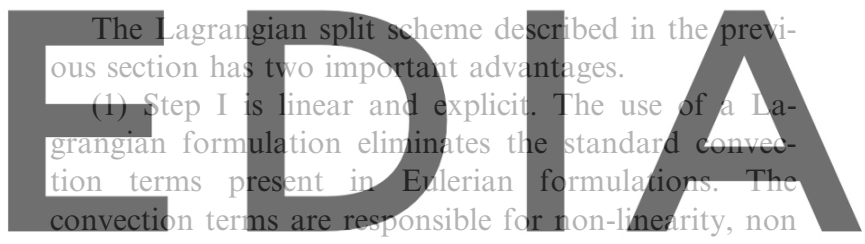

symmetry and non self-adjoint operators which require

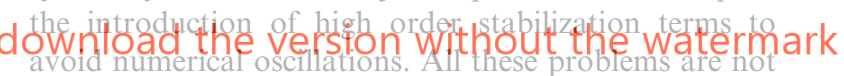
present in this formulation.

(2) In all the five steps described in previous section, the only implicit step is the solution of the Laplacian of pressure (step III). This is a scalar, symmetric and positive definite equation. Then, it is very easy to solve it using an iterative scheme (such as the conjugate gradient method).

The big disadvantage of the Lagrangian formulation is the permanent updating of the node positions. That is the reason why standard finite element methods are not useful, as the process of updating conforming nonstructured finite element meshes is expensive.

The key of the Lagrangian formulation is the efficiency in the mesh updating process. In a previous paper [11], the authors evaluated the use of a meshless method for this purpose. In [11] a meshless method based in point collocation was used. This introduces some difficulty in prescribing the boundary conditions.

Other meshless methods as the element free Galerkin method (EFGM) [5] or the natural element method (NEM) [19] have difficulties to solve arbitrary point distributions in a 3-D domain due to the complicated shape functions used. 
In this paper, the MFEM proposed in Ref. [12] will be used. The method is summarized in Appendix A.

The big advantage of the MFEM compared with the FEM is the possibility of generating meshes in a computing time of order $n$, being $n$ the total number of nodes [14]. Compared with EFGM or NEM, the advantages are the simplicity of the shape functions, which are coincident with the FEM shape functions in most parts of the domain.

Using the MFEM, the unknown functions are approximated using an equal order interpolation for all variables as (in matrix form)

$u_{i}=N_{i}^{\mathrm{T}} U=\left[\begin{array}{lll}N^{\mathrm{T}} & & \\ & N^{\mathrm{T}} & \\ & & N^{\mathrm{T}}\end{array}\right] U$

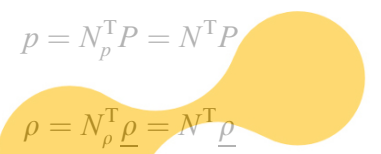

where $N^{\mathrm{T}}$ are the MFEM shape functions and $U, P, \rho$ the nodal values of the three components of the un-

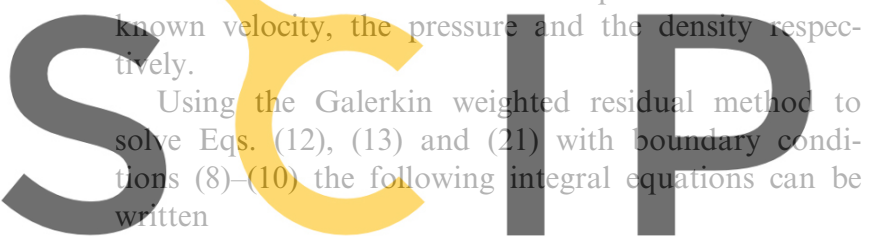

(B) $\frac{1}{\Delta t^{2}} \int_{V} N_{p}\left(\rho^{0}-\rho^{n}\right) \mathrm{d} V$

$$
\begin{aligned}
& -\int_{V} \frac{\partial N_{p}}{\partial x_{i}}\left(\frac{\rho}{\Delta t} u_{i}^{*}-\frac{\partial\left(p^{n+1}-\gamma p^{n}\right)}{\partial x_{i}}\right) \mathrm{d} V \\
& +\frac{\rho}{\Delta t} \int_{\Gamma_{u}} N_{p} \bar{u}_{n}^{n+1} \mathrm{~d} \Gamma=0
\end{aligned}
$$

(C) $\int_{V} N_{i}\left\{\left(u_{i}^{n+1}-u_{i}^{*}\right) \frac{\rho}{\Delta t}+\frac{\partial}{\partial x_{i}}\left(p^{n+1}-\gamma p^{n}\right)\right\} \mathrm{d} V$

$$
-\int_{\Gamma_{\sigma}} N_{i}\left(p^{n+1}-\gamma p^{n}\right) \mathrm{d} \Gamma=0
$$

It must be noted than the essential and natural boundary conditions of equations (29) are

$$
p=0 \quad \text { on } \Gamma_{\sigma}
$$

$$
\bar{u}^{n+1} \cdot v=0 \quad \text { on } \Gamma_{u}
$$

\section{Discrete equations}

Using the approximations (22)-(24) the discrete equations become:

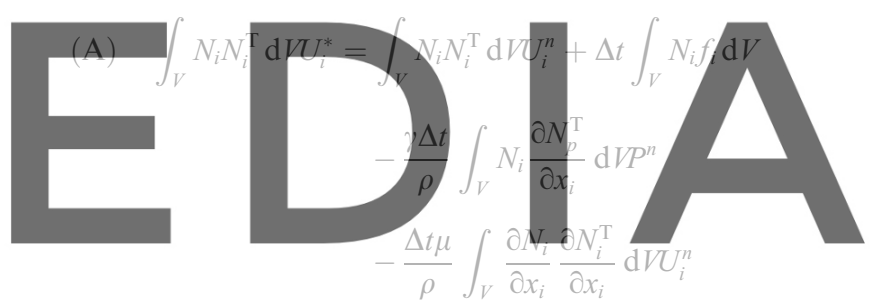

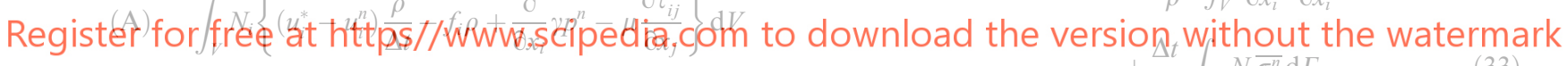

$$
-\int_{\Gamma_{\sigma}} N_{i}\left(\bar{\sigma}_{n i}^{n}-\left(\tau_{i j}^{n} v_{j}-\gamma p^{n} v_{i}\right) \mathrm{d} \Gamma=0\right.
$$

(B) $\int_{V} N_{p}\left\{\frac{\rho^{0}-\rho^{n}}{\Delta t^{2}}+\frac{\rho}{\Delta t} \frac{\partial}{\partial x_{i}} u_{i}^{*}-\frac{\partial^{2}}{\partial x_{i}^{2}}\left(p^{n+1}-\gamma p^{n}\right)\right\} \mathrm{d} V$

$$
+\frac{\rho}{\Delta t} \int_{\Gamma u} N_{p}\left(\bar{u}_{i}^{n+1} v_{i}-u_{i}^{n+1} v_{i}\right) \mathrm{d} \Gamma=0
$$

(C) $\int_{V} N_{i}\left\{\left(u_{i}^{n+1}-u_{i}^{*}\right) \frac{\rho}{\Delta t}+\frac{\partial}{\partial x_{i}}\left(p^{n+1}-\gamma p^{n}\right\} \mathrm{d} V\right.$

$$
-\int_{\Gamma_{\sigma}} N_{i}\left(p^{n+1}-\gamma p^{n}\right) v_{i} \mathrm{~d} \Gamma=0
$$

where the boundary conditions have been also split.

Integrating by parts some of the terms, the above equations become

$$
\begin{gathered}
\int_{V} N_{i}\left(u_{i}^{*}-u_{i}^{n}-f_{i} \Delta t\right) \frac{\rho}{\Delta t} \mathrm{~d} V+\int_{V} N_{i} \frac{\partial}{\partial x_{i}} \gamma p^{n} \\
+\mu \int_{V} \frac{\partial N_{i}}{\partial x_{i}} \frac{\partial u_{i}^{n}}{\partial x_{i}} \mathrm{~d} V-\int_{\Gamma \sigma} N_{i} \bar{\sigma}_{n i}^{n} \mathrm{~d} \Gamma=0
\end{gathered}
$$

In compact form

(A) $M_{u} U^{*}=M_{u} U^{n}+\Delta t F-\frac{\gamma \Delta t}{\rho} B^{\mathrm{T}} P^{n}-\frac{\Delta t \mu}{\rho} K U^{n}$

In the same way

$$
\text { (B) } \begin{aligned}
\frac{1}{\Delta t^{2}} & \left(\int_{V} N_{p} N_{p}^{\mathrm{T}} \mathrm{d} V \underline{\rho}^{0}-\int_{V} N_{p} N_{\rho}^{\mathrm{T}} \mathrm{d} V \underline{\rho}^{n}\right) \\
& -\frac{\rho}{\Delta t} \int_{V}\left(\frac{\partial N_{p}}{\partial x_{i}} N_{i}^{\mathrm{T}}\right) \mathrm{d} V U^{*}+\frac{\rho}{\Delta t} \int_{\Gamma_{u}} N_{p} \bar{u}_{n}^{n+1} \mathrm{~d} \Gamma \\
& =-\int_{V}\left(\frac{\partial N_{p}}{\partial x_{i}} \frac{\partial N_{p}^{\mathrm{T}}}{\partial x_{i}}\right) \mathrm{d} V\left(P^{n+1}-\gamma P^{n}\right)
\end{aligned}
$$

In compact form

(B) $-\frac{M_{p}\left(\underline{\rho}^{0}-\underline{\rho}^{n}\right)}{\Delta t^{2}}+\frac{\rho}{\Delta t} B U^{*}-\frac{\rho}{\Delta t} \widehat{U}+S \gamma P^{n}=S P^{n+1}$ 
and

$$
\text { (C) } \begin{aligned}
& \int_{V} N_{i} N_{i}^{\mathrm{T}} \mathrm{d} V U^{n+1} \\
= & \int_{V} N_{i} N_{i}^{\mathrm{T}} \mathrm{d} V U^{*}-\frac{\Delta t}{\rho} \int_{V} N_{i} \frac{\partial N_{p}^{\mathrm{T}}}{\partial x_{i}} \mathrm{~d} V\left(P^{n+1}-\gamma P^{n}\right) \\
& +\int_{\Gamma_{\sigma}} N_{i} N_{p}^{\mathrm{T}} \mathrm{d} \Gamma\left(P^{n+1}-\gamma P^{n}\right)
\end{aligned}
$$

In compact form (noting that $p=0$ on $\Gamma_{\sigma}$ )

(C) $M_{u} U^{n+1}=M_{u} U^{*}-\frac{\Delta t}{\rho} B^{\mathrm{T}}\left(P^{n+1}-\gamma P^{n}\right)$

where the matrices are

$$
M=\left[\begin{array}{ccc}
M_{p} & 0 & 0 \\
0 & M_{p} & 0 \\
0 & 0 & M_{p}
\end{array}\right]
$$

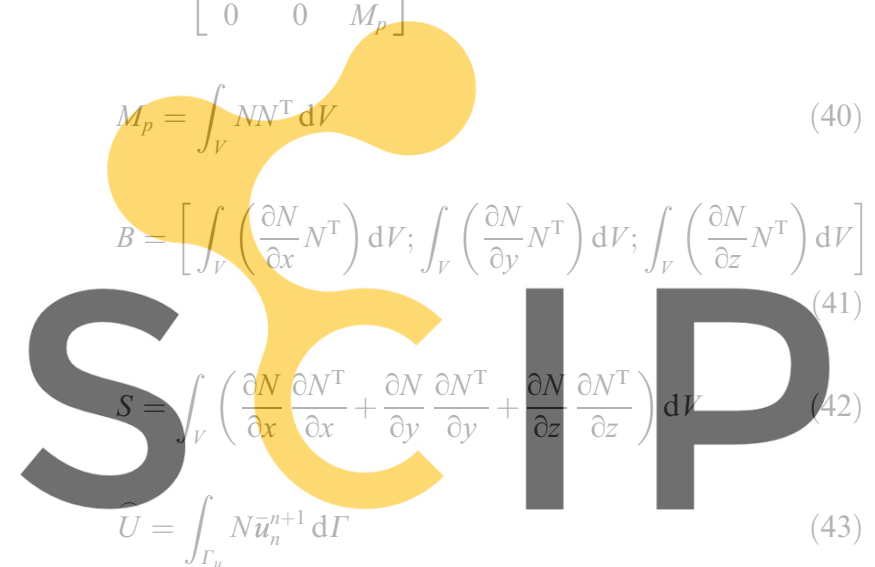

\section{Register for free at https//www.scipedia.com to}

$$
\begin{aligned}
K= & {\left[\begin{array}{ccc}
S & 0 & 0 \\
0 & S & 0 \\
0 & 0 & S
\end{array}\right] } \\
F^{\mathrm{T}}= & {\left[\int_{V} N^{\mathrm{T}} f_{x} \mathrm{~d} V ; \quad \int_{V} N^{\mathrm{T}} f_{y} \mathrm{~d} V ; \quad \int_{V} N^{\mathrm{T}} f_{z} \mathrm{~d} V\right] } \\
& +\frac{1}{\rho}\left[\int_{V} N^{\mathrm{T}} \bar{\sigma}_{n x} \mathrm{~d} V ; \quad \int_{V} N^{\mathrm{T}} \bar{\sigma}_{n y} \mathrm{~d} V ; \quad \int_{V} N^{\mathrm{T}} \bar{\sigma}_{n z} \mathrm{~d} V\right]
\end{aligned}
$$

\section{Stabilization of the incompressibility condition}

In the Eulerian form of the momentum equations, the discrete form must be stabilized in order to avoid numerical wiggles in the velocity and pressure results. This is not the case in the Lagrangian formulation where no stabilization parameter must be added in equations (34) and (38). Nevertheless, the incompressibility condition must be stabilized in equal-order approximations to avoid possible pressure oscillations.

Then, Eq. (36) must be stabilized if smooth pressure results are important. It must be noted than pressure oscillations do not influence significantly in the velocity results. Nevertheless, in most physical problems, pressure is the main result to be obtained. That is why stabilization of Eq. (36) must be performed.

The so-called finite calculus (FIC) formulation [2022] will be chosen here as the stabilization procedure. This formulation is based in the modification of the governing differential equations of the problem by accepting that the domain where the balance laws are established (balance of momentum and balance of mass) has a finite size. The modified equations in the FIC formulation for incompressible fluids are momentum

$r_{i}-\frac{h_{k}}{2} \frac{\partial r_{i}}{\partial x_{k}}=0$

mass conservation

$r-\frac{h_{k}}{2} \frac{\partial r}{\partial x_{k}}=0$

where from Eqs. (1) and (2) the residuals are defined by $r_{i}=\rho \frac{\mathrm{D} u_{i}}{\mathrm{D} t}+\frac{\partial p}{\partial x_{i}}-\frac{\partial \tau_{i j}}{\partial x_{j}}-\rho f_{i}$ (48)

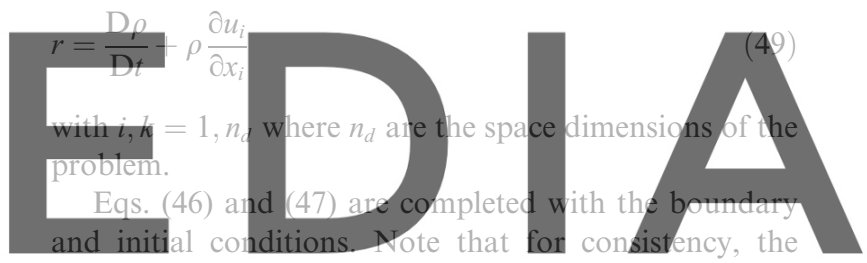

Neumann boundary condition on $\Gamma_{\sigma}$ must also be adedouately madified by adding a residual term. The details ark

The underlined terms in Eqs. (46) and (47) introduce

the necessary stabilization in the numerical solution using whatever discretization method. Examples of the application of the FIC approach the convection-diffusion problems and incompressible problems in solids and fluid mechanics are presented in [21,22].

Distances $h_{i}$ in Eqs. (46) and (47) are "characteristic length" parameters and their values control the relevance of the stabilization terms. The computation of the characteristic lengths is a critical issue in the stabilization process [20].

The new terms in the momentum and mass conservation equations stabilize the numerical solution in presence of high values of the convective terms and incompressibility zones, respectively. Obviously, in Lagrangian flows, as in incompressible solid mechanics problems, the relevant stabilization term is that of Eq. (47), as the convective terms are zero in the momentum equations.

For the practical application of the FIC formulation the stabilization term in the mass balance equation is expressed as a function of the residual of the momentum equations using Eq. (46) as 
$\frac{h_{k}}{2} \frac{\partial r}{\partial x_{k}} \cong \sum_{i=1}^{n_{d}} \tau_{i} \frac{\partial r_{i}}{\partial x_{i}}$

where $\tau_{i}$ are intrinsic time parameters given by

$\tau_{i}=\frac{3 h_{i}^{2}}{8 \mu}$

The modified incompressibility equation is therefore written for the numerical computations as

$r-\sum_{i=1}^{n_{d}} \tau_{i} \frac{\partial r_{i}}{\partial x_{i}}=0$

The stabilization terms in the momentum Eq. (46) are dropped here onwards for the numerical solution.

It is convenient to rewrite the residual $r_{i}$ in Eq. (48) as

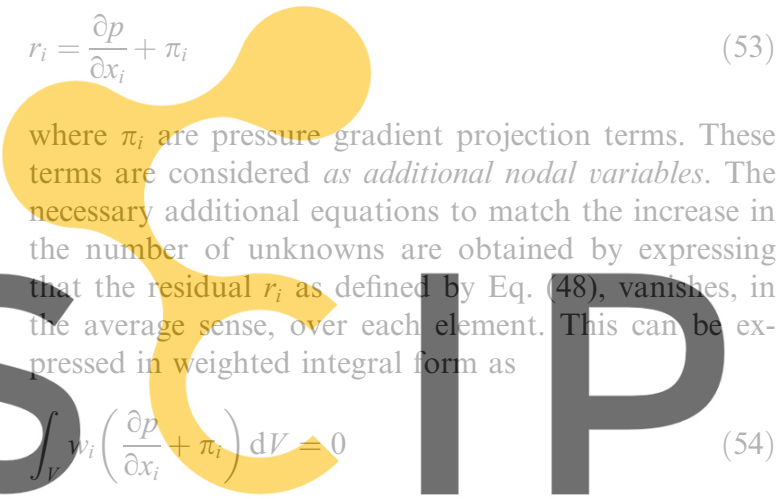

where $w_{i}$ are appropriate weighting functions.

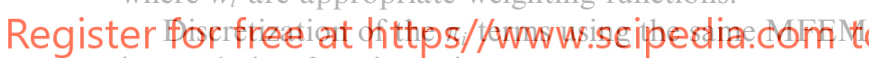

interpolation functions gives

$\pi_{i}=N_{i}^{\mathrm{T}} \Pi$

where $\Pi$ represents the local value of the three components of the pressure gradient. Eq. (54) leads to an equation system of the form (for $w_{i}=N_{i}$ )

$M \Pi+B^{\mathrm{T}} P=0$

Eq. (21) is now modified with the new stabilization term as

$\frac{\rho^{0}-\rho^{n}}{\Delta t^{2}}+\frac{\rho}{\Delta t} \frac{\partial u_{i}^{*}}{\partial x_{i}}=\frac{\partial^{2}}{\partial x_{i}^{2}}\left(p^{n+1}-\gamma p^{n}\right)+\sum_{i=1}^{n_{d}} \frac{\tau_{i}}{\Delta t} \frac{\partial r_{i}}{\partial x_{\mathrm{i}}}$

and Eq. (26) becomes now

$$
\begin{gathered}
\int_{V} N_{p}\left\{\frac{\rho^{0}-\rho^{n}}{\Delta t^{2}}+\frac{\rho}{\Delta t} \frac{\partial}{\partial x_{i}} u_{i}^{*}-\frac{\partial^{2}}{\partial x_{i}^{2}}\left(p^{n+1}-\gamma p^{n}\right)\right. \\
\left.-\sum_{i=1}^{n_{d}} \frac{\tau_{i}}{\Delta t} \frac{\partial r_{i}}{\partial x_{i}}\right\} \mathrm{d} V+\text { boundary terms }
\end{gathered}
$$

Integrating by parts, the equivalent to Eq. (29) is

$$
\begin{aligned}
& \frac{1}{\Delta t^{2}} \int_{V} N_{p}\left(\rho^{0}-\rho^{n}\right) \mathrm{d} V-\int_{V} \frac{\partial N_{p}}{\partial x_{i}}\left\{\frac{\rho}{\Delta t} u_{i}^{*}-\frac{\partial\left(p^{n+1}-\gamma p^{n}\right)}{\partial x_{i}}\right. \\
& \left.-\sum_{i=1}^{n_{d}} \frac{\tau_{i}}{\Delta t}\left(\frac{\partial p^{n+1}}{\partial x_{i}}+\pi_{i}^{n+1}\right)\right\} \mathrm{d} V+\text { b.t. }=0
\end{aligned}
$$

Introducing the discretization of the different fields, and using a compact notation gives

$$
\begin{aligned}
& -\frac{M_{p}\left(\underline{\rho}^{0}-\underline{\rho}^{n}\right)}{\Delta t^{2}}+\frac{\rho}{\Delta t} B U^{*}-\frac{\rho}{\Delta t} \widehat{U}+S \gamma P^{n}-B_{\tau} \Pi^{n} \\
& \quad=\left(S+S_{\tau}\right) P^{n+1}
\end{aligned}
$$

where the new stabilization matrices $B_{\tau}$ and $S_{\tau}$ are defined by
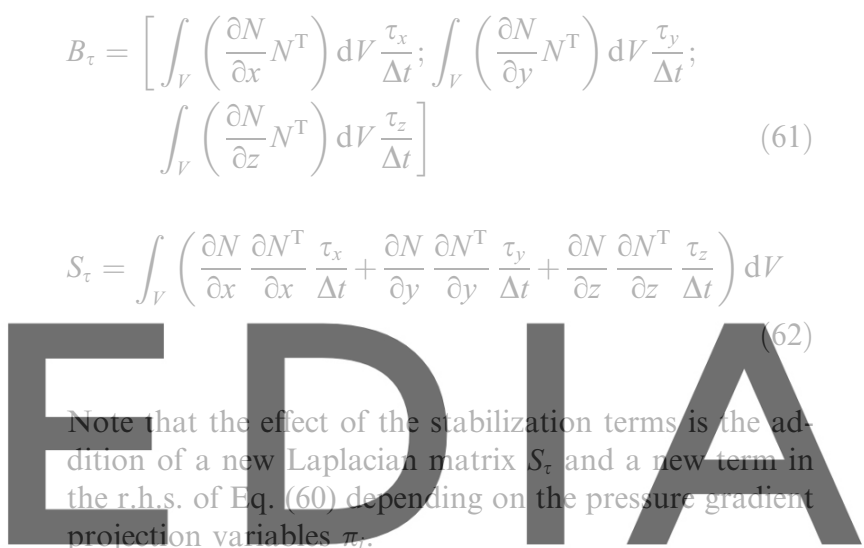

The pressure gradient projection may be evaluated downlicitydusing Eversion without the watermark
(D) $\Pi^{n+1}=-M^{-1} B^{\mathrm{T}} P^{n+1}$

The three steps (A)-(C) described before are now completed with a fourth step (D) where the lumped diagonal form of matrix $M$ may be used.

\section{Mass conservation}

In a Lagrangian formulation a new mesh is generated at each time step, and all the information is transmitted with the nodes or particles. In that way, a local variation in the volume associated with the particles is used as the correct volume in the next time step. A permanent update of the initial volume is necessary to avoid large error accumulation.

Thus, the correct evaluation of the first term of Eq. (36) becomes important in a Lagrangian formulation and will be discussed below.

The term

$M_{p}\left(\underline{\rho}^{0}-\underline{\rho}^{n}\right)$

may be evaluated in two different ways. 
(I) Evaluation via a density update

From the mass conservation equation, the density at time $t^{n}$ may be computed as

$\rho^{n}=\rho^{n-1}-\rho \Delta t \frac{\partial u_{i}^{n}}{\partial x_{i}}$

Making use of the spatial discretization (22) and (24) and the Galerkin residual method gives

$\int_{V} N_{p} N_{p}^{\mathrm{T}} \mathrm{d} V \underline{\rho}^{n}=\int_{V} N_{p} N_{p}^{\mathrm{T}} \mathrm{d} V \underline{\rho}^{n-1}-\rho \Delta t \int_{V} N_{p} \frac{\partial N_{i}^{\mathrm{T}}}{\partial x_{i}} \mathrm{~d} V U^{n}$

Integrating by parts the last term

$\int_{V} N_{p} N_{p}^{\mathrm{T}} \mathrm{d} V \underline{\rho}^{n}=\int_{V} N_{p} N_{p}^{\mathrm{T}} \mathrm{d} V \underline{\rho}^{n-1}+\rho \Delta t \int_{V} \frac{\partial N_{p}^{\mathrm{T}}}{\partial x_{i}} N_{i}^{\mathrm{T}} \mathrm{d} U U^{n}$ $\rho \Delta t \int_{p} \overline{u_{n}^{n}} \mathrm{~d} \Gamma$

or in compact notation

$M_{p} \underline{\rho}^{n}=M_{p} \underline{\rho}^{n-1}+\rho \Delta t B U^{n}-\rho \Delta t \hat{U}$

In order to take into account that the shape functions $N$ are different at each mesh update

will be used: the shape function

ated at the time $t^{n}$ will be noted

(68) becomes

a. $=M_{p}^{n} \rho^{n-1}+\rho \Delta^{n} t B^{n} U^{n}-\rho \Delta t \hat{U}^{n}$

(69)
$\underline{\Delta^{l} \rho}=\rho\left\{\Delta^{l} t\left(M_{p}^{l}\right)^{-1} B^{l} U^{l}-\Delta^{l} t \hat{U}^{l}\right\}$

must be evaluated, added to the previous one and stored for the next time step.

(II) Evaluation via the initial associated volume mass conservation implies

$\int_{V_{(t=0)}} \rho^{0} \mathrm{~d} V=\int_{V_{(t=n)}} \rho^{n} \mathrm{~d} V$

Using the shape functions at the corresponding time step

$\int_{V_{(t=0)}}\left(N_{\rho}^{0}\right)^{\mathrm{T}} \mathrm{d} V \underline{\rho}^{0}=\int_{V_{(t=n)}}\left(N_{\rho}^{n}\right)^{\mathrm{T}} \mathrm{d} V \underline{\rho}^{n}$

Defining the volume associated to each particle by

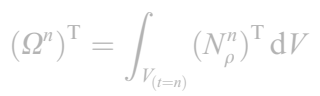

Eq. (76) becomes

$\left(\Omega^{0}\right)^{\mathrm{T}} \underline{\rho}^{0}=\left(\Omega^{n}\right)^{\mathrm{T}} \underline{\rho}^{n}$

which has the meaning of the total mass conservation. Vector $\Omega^{n}$ may be considered as the vector containing the volumes associated to ach particle. It may pe cal-
culated using (77) or using the Voronoi diagrand of the
node distribution.
The concept of local mass conservation my be used
next. This means that each particle (node) conserves his
own local mas i.

where $\Delta^{n} t$ represents the time incremental time $t^{n}$

Registenffor free at https//www.s

$$
\begin{aligned}
\rho^{n} & =\underline{\rho}^{n-1}+\rho \Delta^{n} t\left(M_{p}^{n}\right)^{-1} B^{n} U^{n}-\rho \Delta t \hat{U}^{n} \\
& =\underline{\rho}^{n-1}+\underline{\Delta^{n} \rho}
\end{aligned}
$$

where the density variation has been defined by

$\underline{\Delta^{n} \rho}=\rho \Delta^{n} t\left(M_{p}^{n}\right)^{-1} B^{n} U^{n}-\rho \Delta t \hat{U}^{n}$

representing the $\rho$ variation at time $t^{n}$.

Successive application of Eq. (70) for all time steps gives:

$$
\begin{aligned}
\underline{\rho^{n}} & =\underline{\rho}^{0}+\sum_{l=1}^{n} \underline{\Delta^{l} \rho} \\
& =\underline{\rho}^{0}+\rho \sum_{l=1}^{n}\left\{\Delta^{l} t\left(M_{p}^{l}\right)^{-1} B^{l} U^{l}-\Delta^{l} t \hat{U}^{l}\right\}
\end{aligned}
$$

The term $M_{p}\left(\underline{\rho}^{0}-\underline{\rho}^{n}\right)$ of the r.h.s. of Eq. (36) can be written as

$M_{p}\left(\underline{\rho}^{0}-\underline{\rho}^{n}\right)=-M_{p} \sum_{l=1}^{n} \underline{\Delta^{l} \rho}$

This means that at each time step $t^{l}$, the vector

\section{to downio $\Omega^{0} \Omega^{n} \mathbb{d}^{n}$ the version without the watermark}

The term $M_{p}\left(\rho^{0}-\rho^{n}\right)$ may be written as

$\underline{\underline{\Omega}}^{n}\left(\underline{\rho}^{0}-\underline{\rho}^{n}\right)=\underline{\underline{\Omega}}^{n} \underline{\rho}^{0}-\underline{\underline{\Omega}}^{0} \underline{\rho}^{0}=\rho^{0}\left(\underline{\underline{\Omega}}^{n}-\underline{\underline{\Omega}}^{0}\right)$

where $\underline{\underline{\Omega}}^{0}$ and $\underline{\underline{\Omega}}^{n}$ represent a diagonal matrix with the volume associated to each particle at time $t=t^{0}$ and $t=t^{n}$, respectively.

These matrices may be evaluated using the lumped matrices $M_{\rho}^{0}$ and $M_{\rho}^{n}$ or directly using the associated volume to each particle obtained from a Voronoï diagram.

\section{Boundary surfaces}

One of the main problems in mesh generation is the correct definition of the boundary domain. Sometimes, boundary nodes are explicitly defined as special nodes, which are different from internal nodes. In other cases, the total set of nodes is the only information available and the algorithm must recognize the boundary nodes. Such is the case in the Lagrangian formulation in which, at each time step, a new node distribution is obtained 
and the boundary-surface must be recognized from the node positions.

The use of the MFEM with the extended Delaunay partition makes it easier to recognize boundary nodes.

Considering that the node follows a variable $h(x)$ distribution, where $h(x)$ is the minimum distance between two nodes, the following criterion has been used.

All nodes on an empty sphere with a radius $r(x)$ bigger than $\alpha h(x)$, are considered as boundary nodes.

Thus, $\alpha$ is a parameter close to, but greater than one. Note that this criterion is coincident with the alphashape concept [13].

Once a decision has been made concerning which of the nodes are on the boundaries, the boundary surface must be defined. It is well known that in 3-D problems the surface fitting a number of nodes is not unique. For instance, four boundary nodes on the same sphere may define two different boundary surfaces, a concave one and convex one.
In this work, the boundary surface is defined with all the polyhedral surfaces having all their nodes on the boundary and belonging to just one polyhedron. See Ref. [12].

The correct boundary surface may be important to define the correct normal external to the surface. Furthermore; in weak forms (Galerkin) a correct evaluation of the volume domain is also important. Nevertheless, it must be noted that in the criterion proposed above, the error in the boundary surface definition is of order $h$. This is the standard error of the boundary surface definition in a meshless method for a given node distribution.

\section{Annlication to fluid-structure interactions}

The fluid described above will interact with structures that are in contact with it. Three different cases of
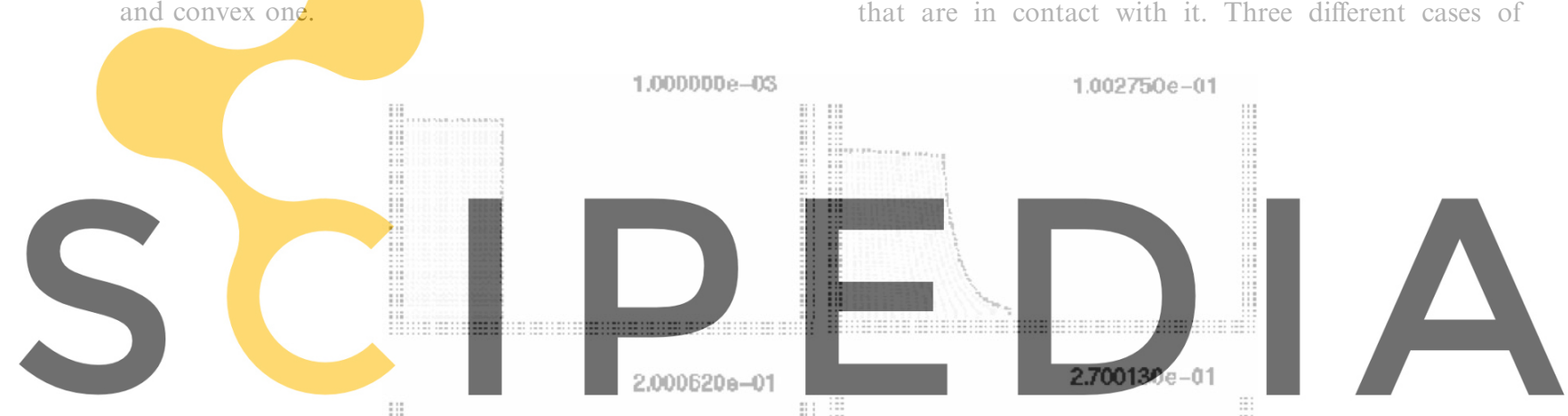

Register for free at https//www.scipedia.com to download the version without the watermark
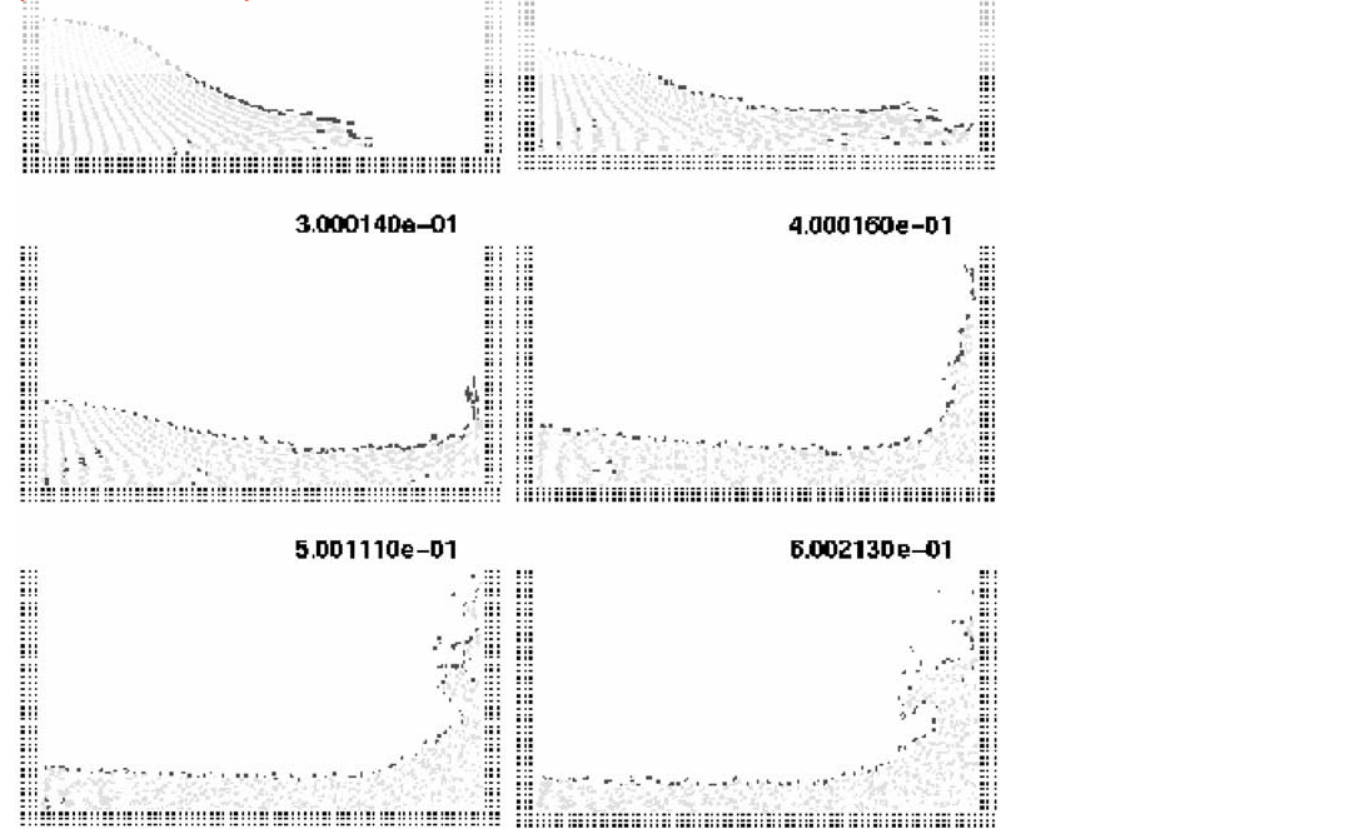

Fig. 1. Water column collapse at different time steps. 
structures will be analyzed. In all three cases, the elastic strains will be neglected and only rigid solid motions will be considered.

\subsection{Fixed structures}

The first type of examples presented is structures in which there is a fixed wall, for instance, the recipient in which the fluid is contained. See Figs. 1 and 2.

This kind of structures will be analyzed by adding fixed particles at the boundaring with velocity $u_{i}=0$. These particles will be included in the computation of equations (A) and (B) as standard nodes, but during equation (C) the velocity will be fixed to zero.

The inclusion of fixed boundary particles is very important to avoid contact problems. These fixed particles automatically force the fluid to remain inside a recipient. The moving particles cannot go across the wall due to the incompressibility condition and not to any other restriction of velocity or displacement. This condition solves the contact problems with complicated curved structures. See for instance example 2.

\subsection{Moving structures with a known velocity}

The second type of FSI is between the fluid and a moving wall of known velocity as a function of the time. This is the case of moving recipients, moving mills, or moving ships with prescribed velocity.

In this case, moving particles with known velocity are introduced in the domain boundaries. Note that the term

\section{$\frac{\rho}{\Delta t} \widehat{U}$

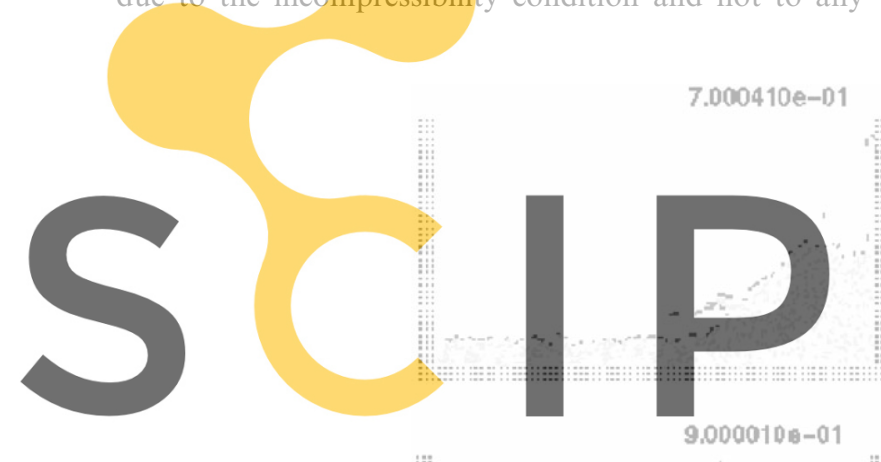

Register for free at https//www.scipedia.com to download the version without the watermark
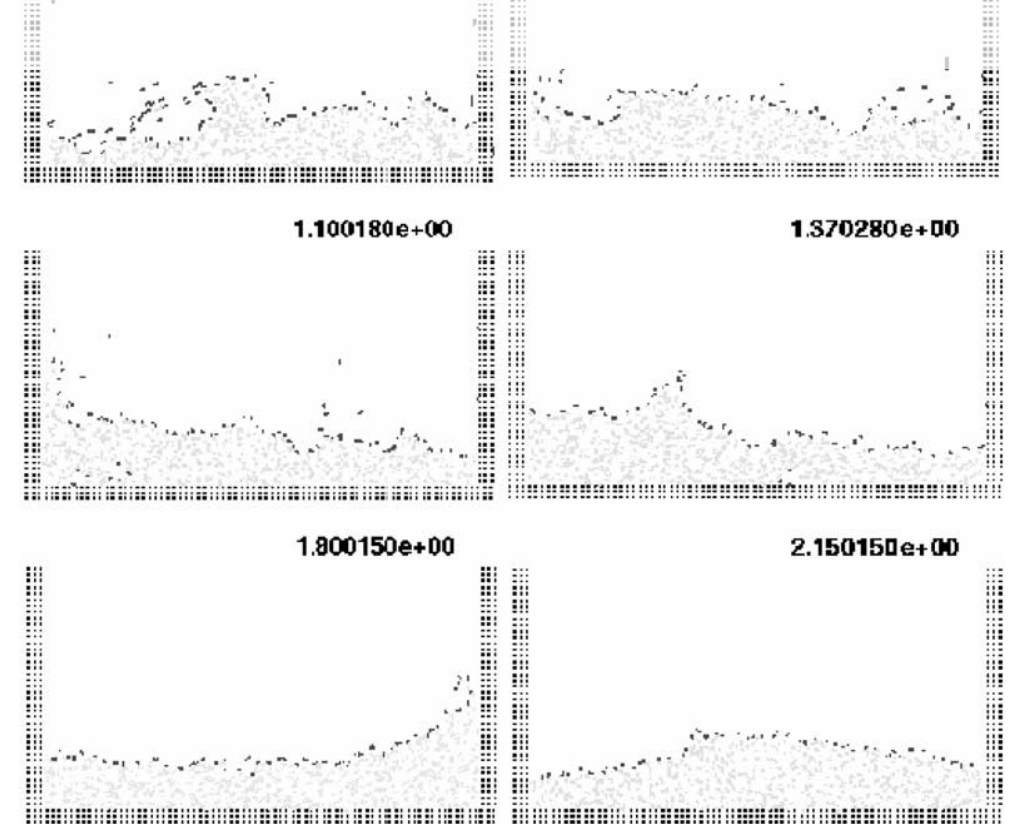

Fig. 1 (continued) 

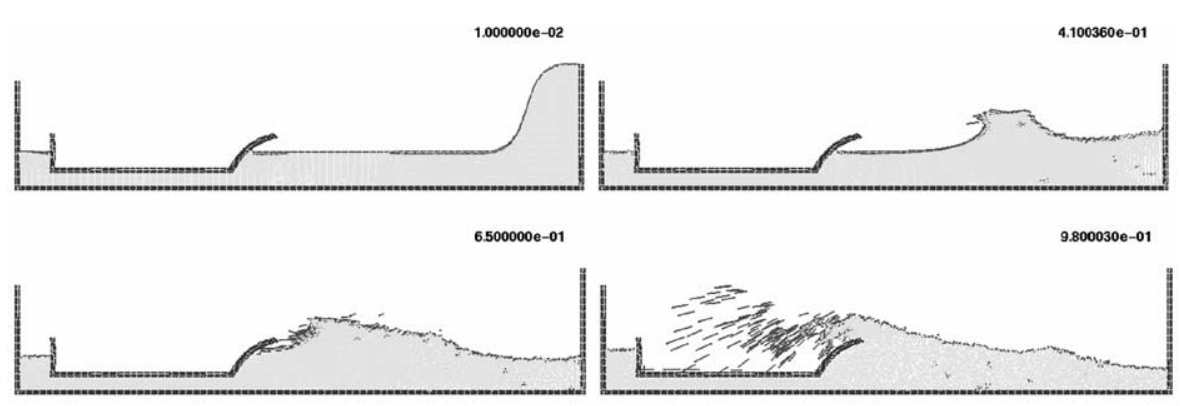

$1.130010 e+00$
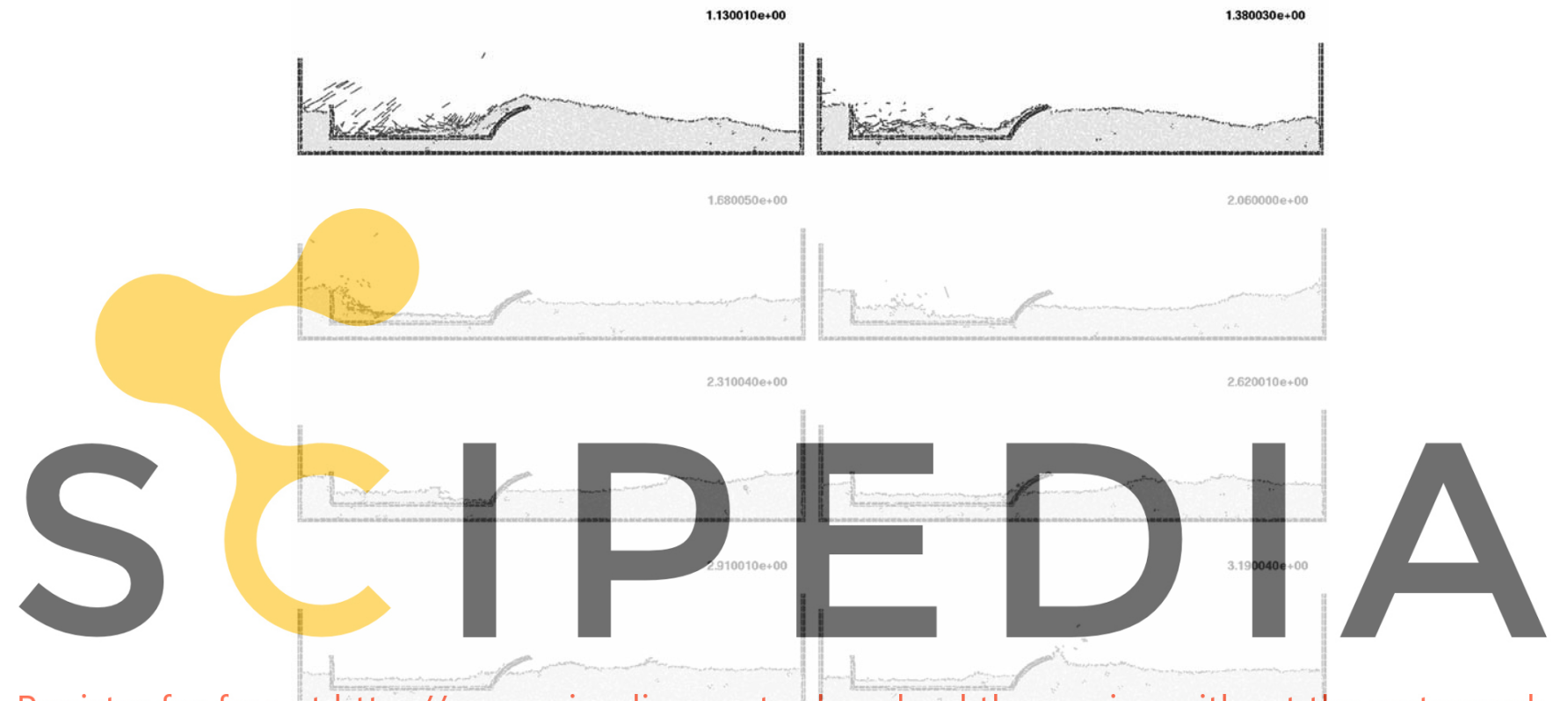

Register for free at https//www.scipedia.com to downtoad the version without the watermark

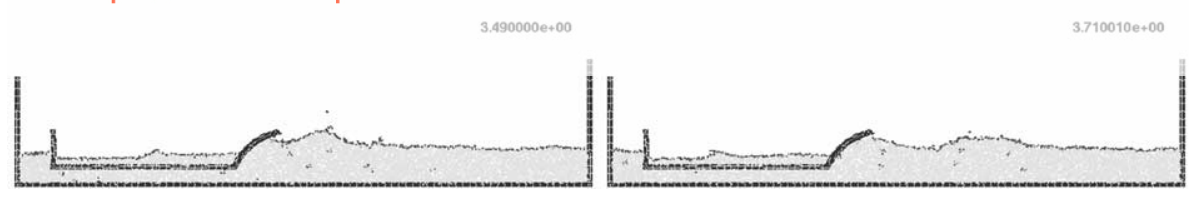

Fig. 2. Fixed ship under external waves.

$\widehat{U}=\int_{\Gamma_{u}} N \bar{u}_{n}^{n+1} \mathrm{~d} \Gamma$

must be added in equation (C) where $\bar{u}_{n}^{n+1}$ are the known velocity on the boundaries. See for instance Figs. 4 and 5 .

\subsection{Moving structures}

Finally, the case of moving rigid structures is considered. For instance, the case of a floating ship (see keeping). In this case, the solid will be considered as a domain with a high viscosity parameter, much higher than the fluid domain. For practical problems a value of
$10^{4} \mu$ is enough to represent a solid without introducing numerical problems (see Figs. 5 and 6).

\section{Numerical test}

\subsection{Water column collapse}

This problem was solved by Koshizu and Oka [4] both experimentally and numerically. It became a classical example to test the validation of the Lagrangian formulation in fluid flows. The water is initially located on the left supported by a removable board. The collapse starts at time $t=0$, when the removable 
board is slid-up. Viscosity and surface tension are neglected.

Fig. 1 shows the point positions at different time steps. The dark points represent the free-surface detected with the alpha-shape algorithm with an alpha parameter $\alpha=1.1$. The internal points are gray and the fixed points are black.

The water is running on the bottom wall until, near $0.3 \mathrm{~s}$, it impinges on the right vertical wall. Breaking waves appear at $0.6 \mathrm{~s}$. Around $1 \mathrm{~s}$, the water reaches the left wall. Agreement with the experimental results of Ref. [4] both in the shape of the free surface as well as in the time development are excellent.

\subsection{Fixed ship under external waves}

This example is a very schematic representation of a ship when it is hit by an external wave (Fig. 2). The ship cannot move and initially the free surface is horizontal with a rectangle on the right wall to produce a big wave. Fixed nodes represent the ship as well as the wall recipient.

The example was created in order to test the suitability of the method to solve contact problems with curved walls correctly. It is interesting to see the crash of the waves under the ship prow and the rebound of the wave on $3.15 \mathrm{~s}$. It is also interesting to see the different
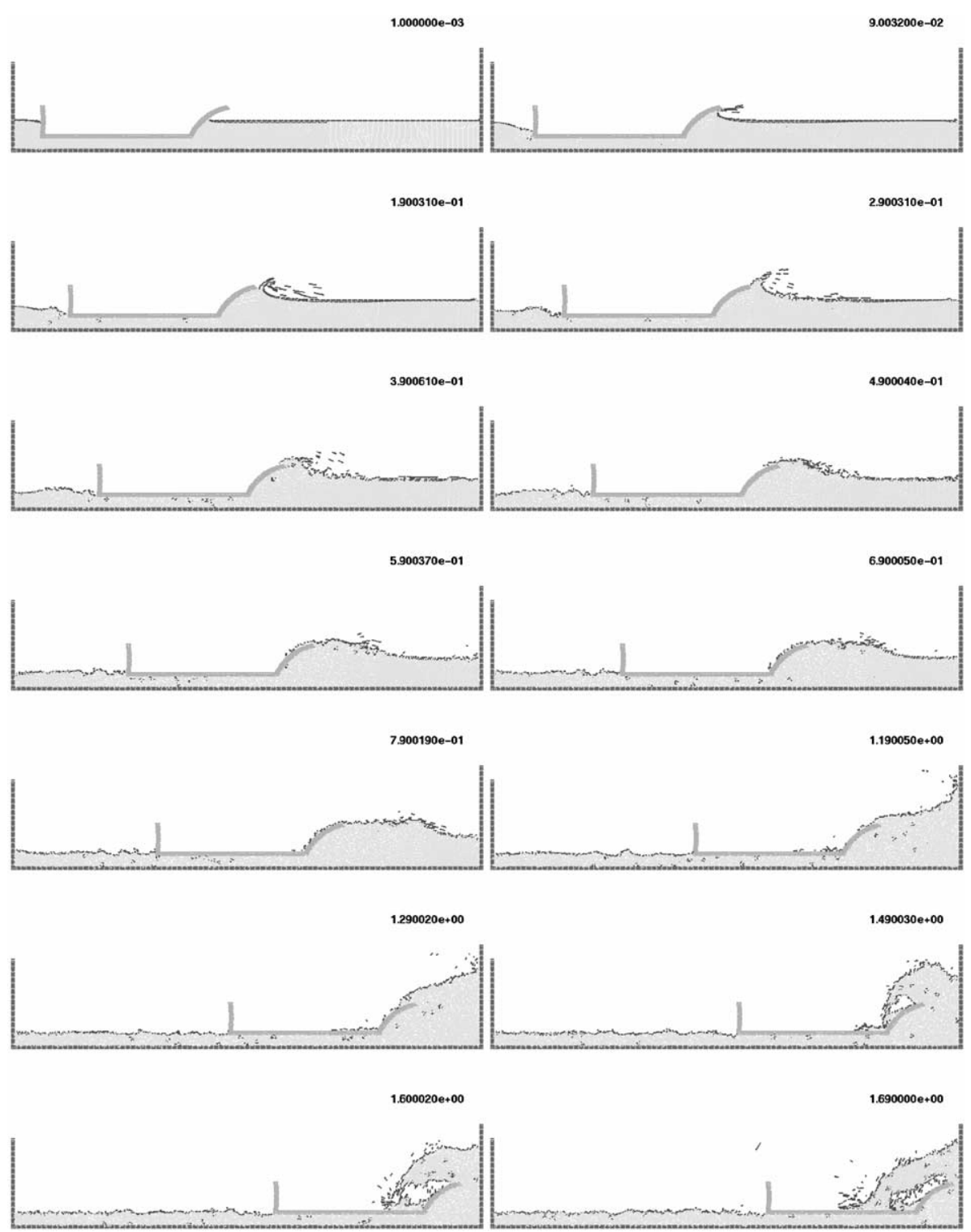

Fig. 3. Moving ship with known velocity. 
contact walls with the internal and external ship surfaces and the moving free surface at the bottom of the ship.

\subsection{Moving ship with known velocity}

In this case (Fig. 3), the same ship of the previous example is now moving at a fixed velocity. All the nodes representing the ship have an imposed velocity. The free surface, which was initially horizontal, takes a correct position at the bottom of the ship, and again, the correct contact problem is realistically solved in the curved prow.

\subsection{Rotating water mill}

A schematic representation of a water mill is presented in Fig. 4. The blades of the mill have an imposed rotating velocity, while the water is initially in a stationary and flat position. Fluid structure interactions with free-surfaces and fragmentation are well reproduced in this example.

\subsection{Solid falling into a recipient with water}

In this example the fluid is interacting with a solid that is totally free, without any imposed velocity. Fig. 5 represents a free cube falling down into a recipient full of water. The solid cube was modeled by introducing a high viscosity parameter in the element in the following way: all the polyhedral elements formed by nodes contained in the solid have a high viscosity value. The other elements are inviscid.

The example represents correctly the contact problem when the cube hits the water and also the different speed during the falling process.

\subsection{Solid floating on a free surface}

The last example of Fig. 6 represents a very interesting problem of fluid structure interaction when there is a weak interaction between the fluid and a large rigid deformation of the structure. In this case, there is also a free-surface problem, representing a schematic case of see-keeping in ship hydrodynamics.

The example shows an initially stationary recipient with a floating piece of wood in which a wave is produced on the left side. The wave intercepts the wood piece producing a breaking wave and moving the floating wood.

All the previous examples are only schematic representations of real problems. Only the first example has an experimental reference. The rest are presented here in order to evaluate the suitability of the method to solve problems other methods have difficulties to solve.
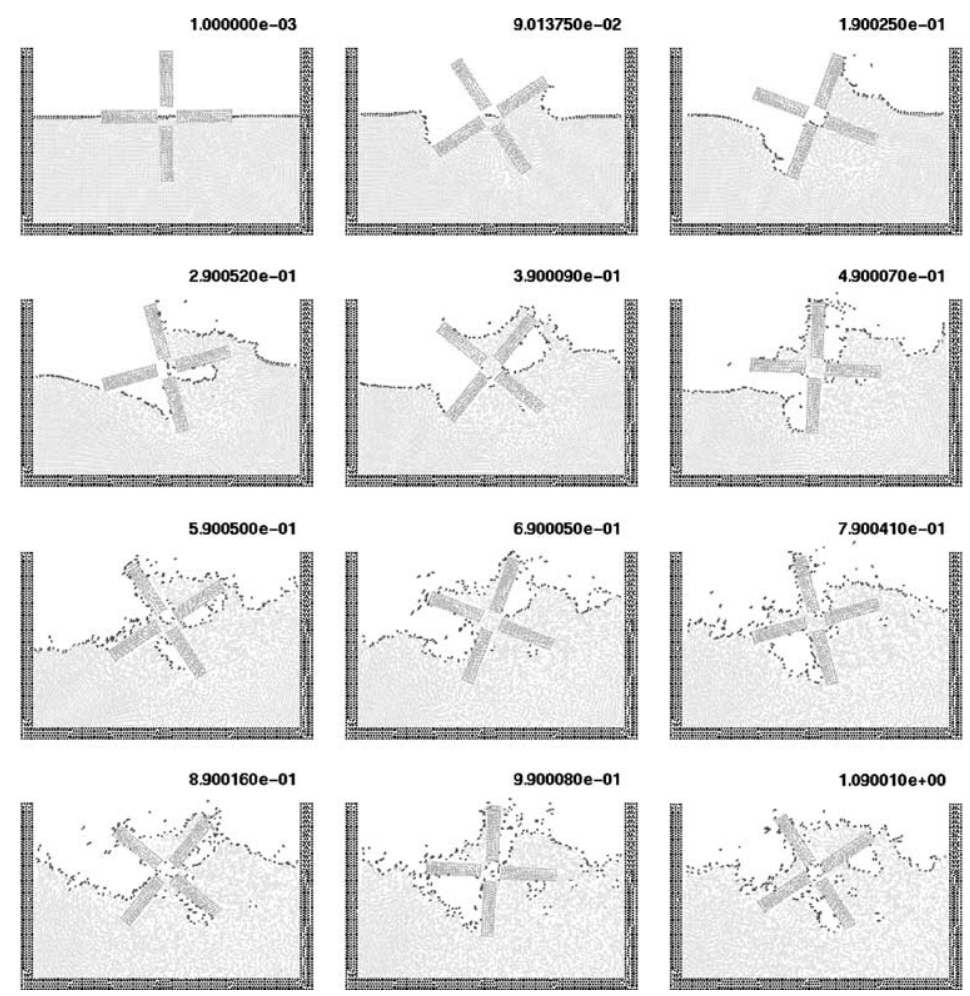

Fig. 4. Rotating water mill. 

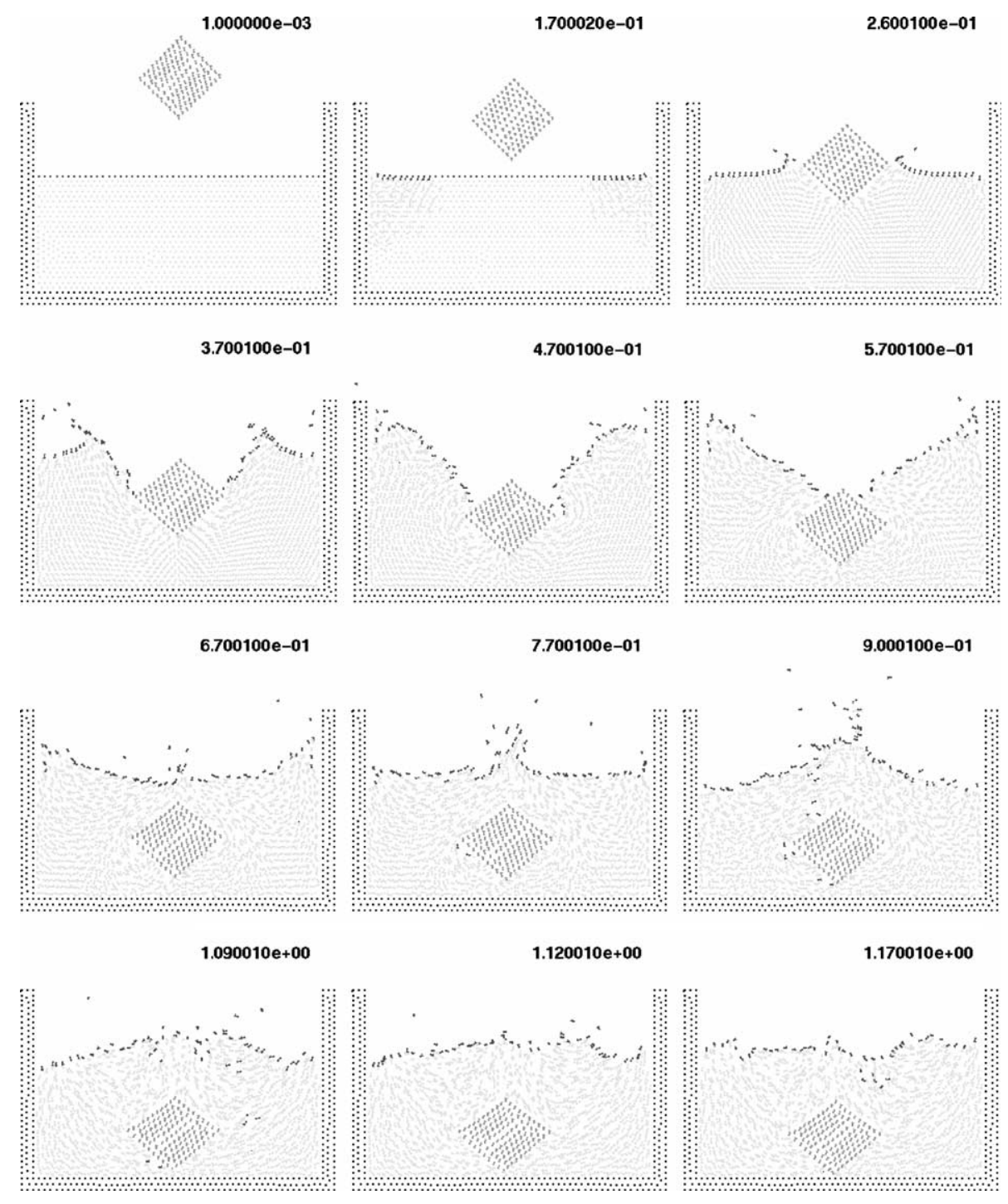

Fig. 5. Solid cube falling into a recipient with water.

\section{Conclusions}

Lagrangian formulation and the MFEM are an excellent combination to solve fluid mechanic problems, especially FSI with moving free-surface and contact problems.

Breaking waves, collapse problems, and contact problems can be solved easily without any additional constraint.

Furthermore, the MFEM presented, as opposed to other methods, has the advantages of a good meshless method concerning the easy introduction of the nodes connectivity in a bounded time of order $n$. The method proposed also shares some advantages with the FEM such as: (a) the simplicity of the shape functions, (b) $C_{0}$ continuity between elements, (c) an easy introduction of the boundary conditions, and (d) symmetric matrices.

The FIC formulation can be successfully used in a Lagrangian formulation in order to eliminate spurious pressure oscillations.

Both the Lagrangian formulation and the MFEM are the key ingredients to solve FSI problems including with free-surface, breaking waves and collapse situations.

\section{Appendix A}

All the shape functions $N_{i}$ described in this paper are based on the MFEM. A full description of the MFEM may be found in Ref. [12]. Nevertheless and for the sake of completeness a summary is presented in this appendix. 
0.01

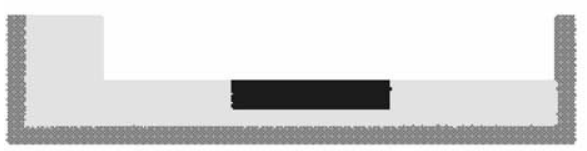

0.58

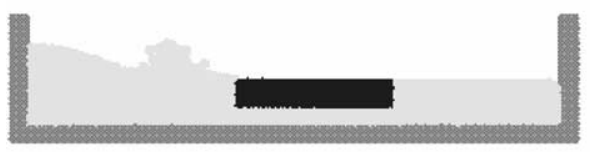

1.18

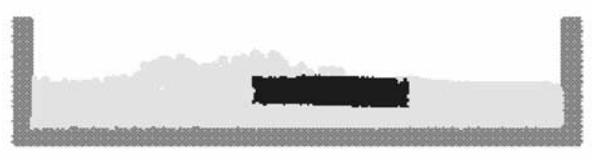

1.78

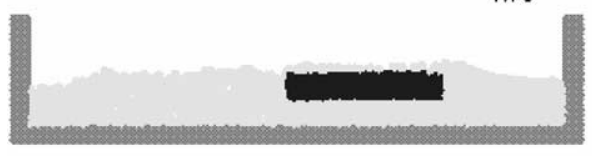

2.38

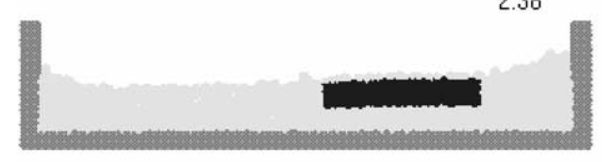

2.98

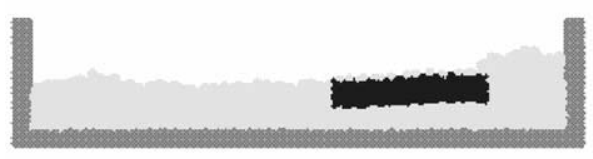

3.58

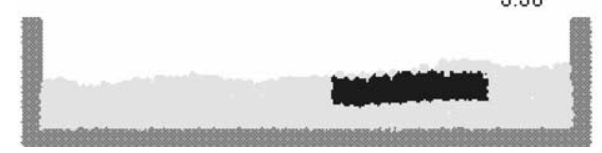

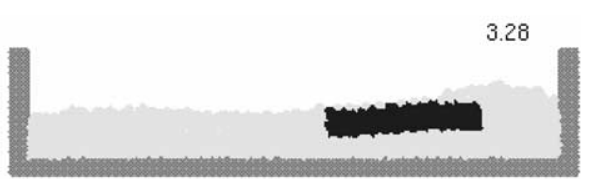

3.88

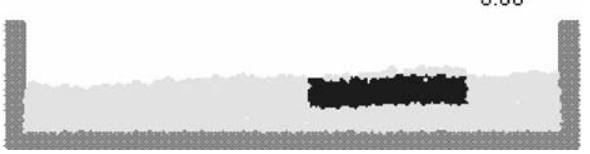

Fig. 6. Solid floating on a free surface.

The MFEM combines a particular finite element subdivision in polyhedral shape called the EDT and ad hoc shape functions for this kind of polyhedra.

\section{A.1. The extended Delaunay tessellation (EDT)} in $R^{3}$.

Let a set of distinct nodes be: $\mathbf{N}=\left\{\mathbf{n}_{1}, \mathbf{n}_{2}, \mathbf{n}_{3}, \ldots, \mathbf{n}_{n}\right\}$

(a) The Voronoi diagram of the set $\mathbf{N}$ is a partition of $R^{3}$ into regions $V_{i}$ (closed and convex, or unbounded), where each region $V_{i}$ is associated with a node $\mathbf{n}_{i}$, such that any point in $V_{i}$ is closer to $\mathbf{n}_{i}$ (nearest neighbor) than to any other node $\mathbf{n}_{i}$. See Fig. 7 for a 2-D representation. There is a single Voronoï diagram for each set $\mathbf{N}$.

(b) A Voronoi sphere within the set $\mathbf{N}$ is any sphere, defined by four or more nodes, that contains no other node inside. Such spheres are also known as empty circumspheres.

(c) A Delaunay tessellation within the set $\mathbf{N}$ is a partition of the convex hull of all the nodes into regions $\Omega_{i}$ such that $\Omega=U \Omega_{i}$, where each $\Omega_{i}$ is the tetrahedron defined by four nodes of the same Voronoï sphere. Delaunay tessellations of a set $\mathbf{N}$ are not unique, but each tessellation is the dual of the single Voronoï diagram of the set. 


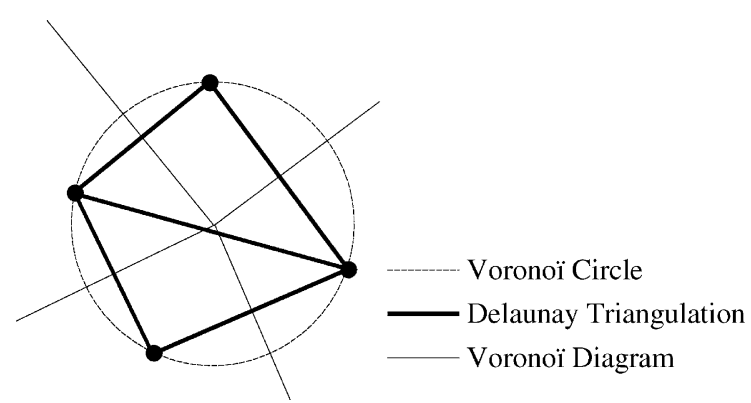

Fig. 7. Voronoï diagram, Voronoï circle and Delaunay triangulation for a four nodes distribution in 2-D.

The computing time required for evaluation of all these three entities is of order $n^{\alpha}$ with $\alpha \leqslant 1.333$. Using a very simple bin organization, the computation time may be reduced to a simple order $n$.

As stated above, the Delaunay tessellation of a set of nodes is non-unique. For the same node distribution, different triangulations (actually tetrahedrations, as it refers to 3-D) are possible. Therefore, an interpolation based on the Delaunay tessellation is sensitive to geometric perturbations of the position of the nodes. On the other hand, its dual, the Voronoï diagram, is unique. Thus, it makes more sense to define meshless shape functions based on the unique Voronoï diagram than on Delaunay tessellations. Furthermore, in 3-D problems the Delaunay tessellation may generate several tetrahedra of zero or almost zero volume, which introduces large inaccuracies into the shape function derivatives. The time to obtain a good mesh via a Delaunay tessellation becomes then an unbounded iterative operation.

These drawbacks appear in the so-called "degenerated case", which is the case where more than four nodes (or more than three nodes in a 2-D problem) are on the same empty sphere. For instance, when five nodes are on the same sphere, five tetrahedra may be defined satisfying the Delaunay criterion, but some of them may have zero or almost zero volumes, called slivers, as seen in Fig. 8:
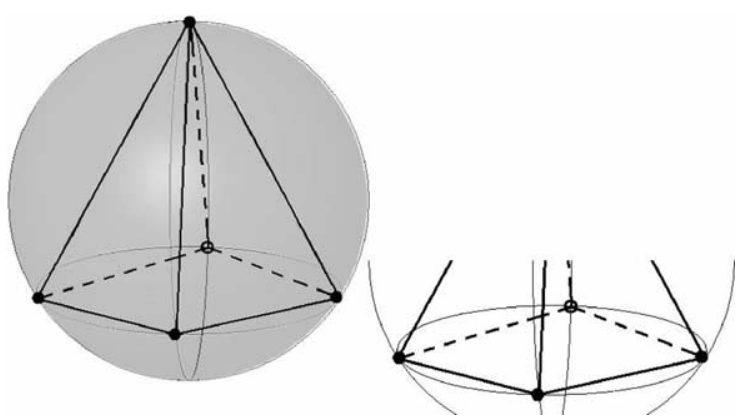

Fig. 8. Five nodes on the same sphere and possible zero or almost zero volume tetrahedron (sliver) on the right.
In order to overcome above drawbacks, a generalization of the Delaunay tessellation will be defined.

Definition. The extended Delaunay tessellation within the set $\mathbf{N}$ is the unique partition of the convex hull $\Omega$ of all the nodes into regions $\Omega_{i}$ such that $\Omega=U \Omega_{i}$, where each $\Omega_{i}$ is the polyhedron defined by all the nodes laying on the same Voronoï sphere.

The main difference between the traditional Delaunay tessellation and the EDT is that, in the latter, all the nodes belonging to the same Voronoi sphere define a unique polyhedron. With this definition, the domain $\Omega$ is divided into tetrahedra and other polyhedra, which are unique for a set of node distributions. Fig. 9 for instance, is a 2-D polygon partition with a triangle, a quadrangle and a pentagon. Fig. 10 is a classical eightnode polyhedron with all the nodes on the same sphere.

For non-uniform node distributions, considering infinite precision, only four nodes are necessary to define a sphere. Other nodes close to the sphere may define other spheres very close to the previous one. In order to avoid this situation, which may hide polyhedra with more than four nodes, a parameter $\delta$ will be introduced. In such a way, the polyhedra are defined by all the nodes of the same sphere and nearby spheres with a distance between center points smaller than $\delta$.

The parameter $\delta$ avoids generating zero volume or near zero volume tetrahedra. When $\delta$ is large, the number of polyhedra with more than four nodes

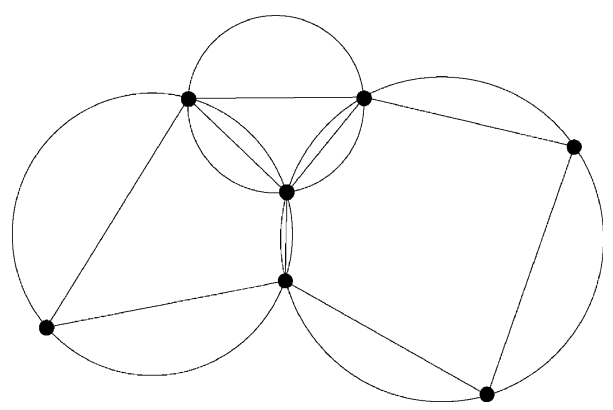

Fig. 9. Two-dimensional partition in polygons. The triangle, the quadrangle and the pentagon are each inscribed on a circle.

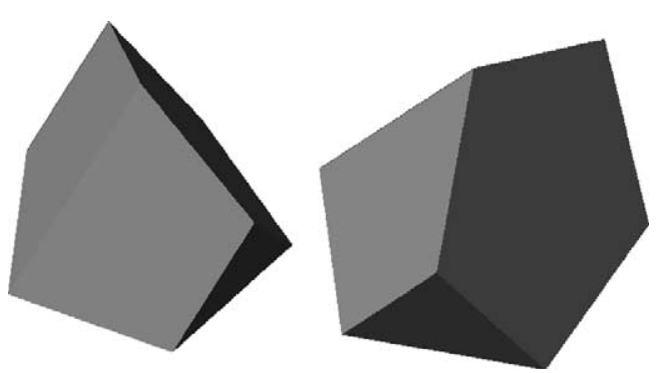

Fig. 10. Eight-node polyhedron. All nodes are on the same sphere. 
increases, and the number of tetrahedra with near zero volume decreases, and vice versa.

The EDT leads to a domain partition which (a) is unique for a set of node distributions; (b) is formed by polyhedra with no zero volume, and (c) is obtained in a bounded time of order $\mathrm{n}$. Then, it satisfies the conditions for a meshless method.

\section{A.2. The meshless finite element shape functions}

Once the domain partition in polyhedra is defined, shape functions must be introduced to solve a discrete problem. In fluid and solid mechanics problems typically, $C^{0}$ continuous shape functions are chosen. If possible, shape functions must be locally supported in order to obtain band matrices. They must also satisfy two criteria in order to have a reasonable convergence order, namely partition of unity and linear completeness.

In order to define the shape functions inside each polyhedron the non-Sibsonian interpolation is used [23].

Let $\mathbf{P}=\left\{\mathbf{n}_{1}, \mathbf{n}_{2}, \ldots, \mathbf{n}_{m}\right\}$ be the set of nodes belonging to a polyhedron. The shape function $N_{i}(\mathbf{x})$ corresponding to the node $\mathbf{n}_{i}$ at an internal point $\mathbf{x}$ is defined by building first the Voronoi cell corresponding to $\mathbf{x}$ in the tessellation of the set $\mathbf{P} U\{\mathbf{x}\}$ and then by computing

$N_{i}(\mathbf{x})=\frac{\frac{s_{i}(\mathbf{x})}{h_{i}(\mathbf{x})}}{\sum_{j=1}^{m} \frac{s_{j}(\mathbf{x})}{h_{j}(\mathbf{x})}}$

where $s_{i}(\mathbf{x})$ is the surface of the Voronoi cell face corresponding to node the node $\mathbf{n}_{i}$ and $h_{i}(\mathbf{x})$ is the distance between point $\mathbf{x}$ and the node $\mathbf{n}_{i}$ (Fig. 11).

Non-Sibsonian interpolations have the following properties [19].

(1)

$$
0 \leqslant N_{i}(\mathbf{x}) \leqslant 1
$$

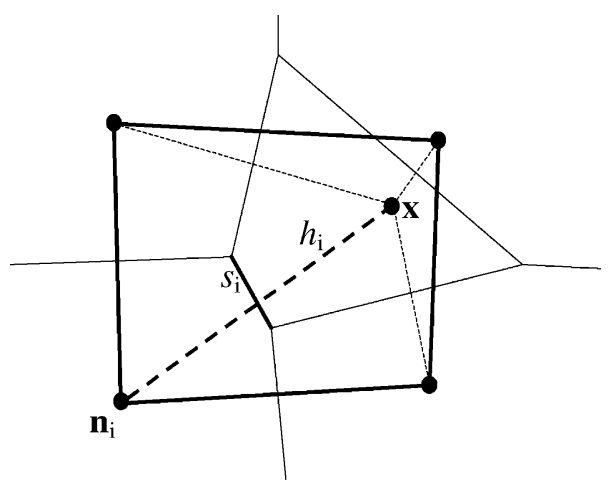

Fig. 11. Four nodes and arbitrary internal point $x$ Voronoï diagram. Shape function parameters.
(2)

$$
\sum_{i} N_{i}(\mathbf{x})=1
$$

(3)

$$
N_{i}\left(\mathbf{n}_{j}\right)=\delta_{i j}
$$

(4)

$$
\mathbf{x}=\sum_{i} N_{i}(\mathbf{x}) \mathbf{n}_{i}
$$

Furthermore, the particular definition of the nonSibsonian shape function for the limited set of nodes on the same Voronoi sphere, adds the following properties.

(5) On a polyhedron surface, the shape functions depend only on the nodes of this surface.

(6) On triangular surfaces (or in all the polygon boundaries in 2-D), the shape functions are linear.

(7) If the polyhedron is a tetrahedron (or a triangle in 2D) the shape functions are the linear finite element shape functions.

(8) Due to property 5 , the shape functions have $C^{0}$ continuity between two neighboring polyhedra. See Fig. 12.

(9) As a matter of fact, because all the element nodes are on the same sphere, the evaluation of the shape functions and its derivatives becomes very simple.

The method MFEM defined here is both a meshless method and a FEM. The algorithm steps for the MFEM are

(1) for a set of nodes, compute all the empty spheres with four nodes;

(2) generate all the polyhedral elements using the nodes belonging to each sphere and the nodes of all the coincident and nearby spheres;

(3) calculate the shape functions and their derivatives, using the non-Sibsonian interpolation, at all the Gauss points necessary to evaluate the integrals of the weak form;

The MFEM is a truly meshless method because the shape functions depend only on the node positions. Furthermore, steps 1 and 2 of the node connectivity process are bounded with $n^{1.33}$, avoiding the mesh "cosmetics" often needed in mesh generators.

The number of Gauss points necessary to compute the element integrals depends, to a great extent, on the polyhedral shape of each element. Note that for an irregular node distribution, there remains a significant amount of tetrahedra (in the examples, more than $85 \%$ of the elements remains tetrahedral) with linear shape functions, for which only one Gauss point is enough. For the remaining polyhedra, the integrals are performed dividing them into tetrahedra and then using a single Gauss point in each tetrahedron. This subdivision 


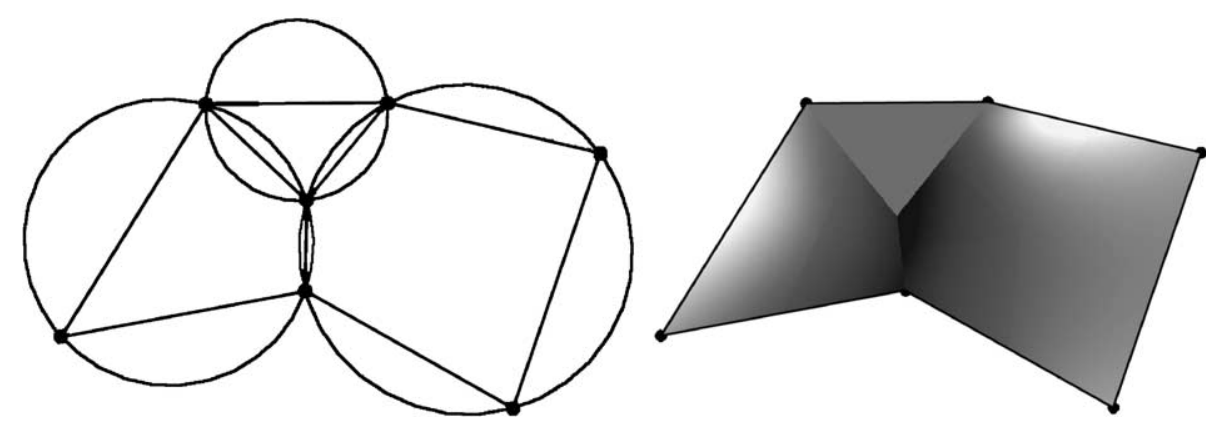

Fig. 12. $C^{0}$ continuity of the shape function on a 2-D node connection.

is only performed for the evaluation of the integrals and cannot be considered as a tetrahedral mesh because it is not conforming. The use of one Gauss point on each tetrahedron guarantee that the computing time in the evaluation of the matrices requires the same effort than the FEM.

\section{References}

[1] Gingold RA, Monaghan JJ. Smoothed particle hydrodynamics, theory and application to non-spherical stars. Mon Nat R Astr Soc 1997;181:375-89.

[2] Bonet J, Kulasegaram S. Convection and stabilization of smooth particle hydrodynamics methods with applications in metal forming simulation. Int J Numer Meth Eng 1999.

[3] Dilts GA. Moving least squares particle hydrodynamics. i. Consistency and stability. Int $\mathbf{J}$ Numer Meth Eng 1999;44:1115-55.

[4] Koshizuka S, Oka Y. Moving particle semi-implicit method for fragmentation of incompressible fluid. Nucl Eng Sci 1996;123:421-34.

[5] Belytschko T, Liu Y, Gu L. Element free Galerkin methods. Int J Numer Meth Eng 1994;37:229-56.

[6] De S, Bathe KJ. The method of finite spheres with improved numerical integration. Comput Struct 2001;79:2183-96.

[7] Nayroles B, Touzot G, Villon P. Generalizing the fem: diffuse approximation and diffuse elements. Computat Mech 1992;10:307-18.

[8] Oñate E, Idelsohn SR, Zienkievicz OC, Taylor RL. A finite point method in computational mechanics. Applications to convective transport and fluid flow. Int J Numer Meth Eng 1996;39(22):3839-86.

[9] Oñate E, Idelsohn SR, Zienkievicz OC, Taylor RL, Sacco C. A stabilized finite point method for analysis of fluid mechanics problems. Comput Meth Appl Mech Eng 1996;39:315-46.

[10] Taylor RL, Zienkiewicz OC, Oñate E, Idelsohn SR, Moving least square approximations for solution of differential equations. Internal report 74, CIMNE, Barcelona, Spain; 1996.

[11] Idelsohn SR, Storti MA, Oñate E. Lagrangian formulations to solve free surface incompressible inviscid fluid flows. Comput Meth Appl Mech Eng 2001;191:583-93.

[12] Idelsohn SR, Oñate E, Calvo N, DelPin F. The meshless finite element method. Int J Numer Meth Eng 2003 [in press].

[13] Edelsbrunner H, Mucke EP. Three-dimensional alphashape. ACM Trans Graph 1994;3:43-72.

[14] Idelsohn SR, Calvo N, Oñate E. Polyhedrization of an arbitrary 3D point set. Comput Meth Appl Mech Eng 2002 [submitted].

[15] Bathe KJ, Zhang H, Ji S. Finite element analysis of fluid flows coupled with structural interactions. Comput Struct 1999;72:1-16.

[16] Zhang H, Bathe KJ. Direct and iterative computing of fluid flows fully coupled with structures. Comput Fluid Solid Mech 2001:1440-3.

[17] Rugonyi S, Bathe KJ. CMES 2001;2(2):195-212.

[18] Codina R. Pressure Stability in fractional step finite element methods for incompressible flows. J Comput Phys 2001;170:112-40.

[19] Sukumar N, Moran B, Semenov AYU, Belikov VV. Natural neighbour Galerkin methods. Int J Numer Meth Eng 2001;50:1-27.

[20] Oñate E. Derivation of stabilized equations for advectivediffusive transport and fluid flow problems. Comput Meth Appl Mech Eng 1998;151(1-2):233-67.

[21] Oñate E. A stabilized finite element method for incompressible viscous flows using a finite increment calculus formulation. Comput Meth Appl Mech Eng 2000;182 (1-2):355-70.

[22] Oñate E. Possibilities of finite calculus in computational mechanics. Int J Num Meth Eng 2002 [submitted].

[23] Belikov V, Semenov, A. Non-Sibsonian interpolation on arbitrary system of points in Euclidean space and adaptive generating isolines algorithm. Numerical grid generation in computational field simulation. In: Proceedings of the 6th international conference Greenwich University, July 1998. 